\title{
O Programa Nacional de Alimentação Escolar sob a ótica dos alunos da rede estadual de ensino de Minas Gerais, Brasil
}

\author{
The Brazilian School Nutrition Program from the standpoint \\ of students attending state schools in Minas Gerais, Brazil
}

Camilo Adalton Mariano da Silva ${ }^{1}$

Luciana Araújo Marques ${ }^{2}$

Élido Bonomo ${ }^{1}$

Olívia Maria de Paula Alves Bezerra ${ }^{1}$

Margareth da Silva Corrêa ${ }^{3}$

Letícia Siqueira Falce Passos ${ }^{2}$

Anelise Andrade de Souza ${ }^{2}$

Betannya França Barros ${ }^{2}$

Débora Maria Soares de Souza ${ }^{2}$

Joana Almeida dos Reis ${ }^{2}$

Noemi Gonçalves de Andrade ${ }^{2}$

\footnotetext{
${ }^{1}$ Departamento de Nutrição Clínica e Social,Escola de Nutrição, Universidade Federal de Ouro Preto. Campus Morro do Cruzeiro S/N. 35400-000 Ouro Preto Minas Gerais. camiloadalton@yahoo.com.br ${ }^{2}$ Centro Colaborador em Alimentação e Nutrição do Escolar,Escola de Nutrição, Universidade Federal de Ouro Preto.

${ }^{3}$ Departamento de Alimentos, Escola de Nutrição, Universidade Federal de Ouro Preto.
}

\begin{abstract}
The scope of this article is to analyze the Brazilian School Nutrition Program from the standpoint of students attending state schools in Minas Gerais. It is a qualitative and quantitative cross-sectional study with a sample of 1500 students, representing the population of the state schools of Minas Gerais, involving the administration of a semi-structured questionnaire. The data were analyzed using simple frequency, mean, standard deviation, Fisher's exact test, the chisquare test and logistic regression $(p<0.05, C I$ 95\%). The effective acceptance of the program was $28.8 \%$ and the effective adhesion to the program was $45.1 \%$. Program acceptance was significantly higher among males and students who consumed less extra-institutional food. Acceptance and adhesion to the program was significantly higher among the older students and those who reported participating in activities related to nutritional education. In total, $73.5 \%$ of the students suggested improvements in school food. Many of the program's norms and guidelines are not being implemented. The acceptance of school food was negatively influenced by the consumption of extra-institutional foods and positively influenced by food and nutritional education activities.
\end{abstract}

Key words Public policies, Health program and project evaluation, School food, Students
Resumo O objetivo deste artigo é analisar o Programa de Alimentação Escolar sob a ótica dos alunos atendidos na rede estadual de ensino de Minas Gerais. Trata-se de estudo transversal, qualiquantitativo, com amostra de 1500 alunos, representativa do universo da rede estadual de ensino de Minas Gerais, utilizando questionário semiestruturado analisado por meio de frequência simples, média, desvio padrão, teste exato de Fisher, teste qui-quadrado e regressão logística $(p<$ 0,05; IC 95\%). Houve efetiva aceitação de 28,8\% e efetiva adesão de 45,1\% ao Programa. A aceitação foi significativamente maior entre alunos do sexo masculino e entre aqueles que apresentaram menor consumo de alimentos extrainstitucionais. A aceitação e a adesão foram significativamente maiores entre alunos do grupo etário mais velho e entre aqueles que referiram participar de atividades de Educação Alimentar e Nutricional. No total, 73,5\% dos alunos sugeriram melhorias para a Alimentação Escolar. Muitos princípios e diretrizes do programa não estão sendo cumpridos. A aceitação da Alimentação Escolar mostrou-se negativamente influenciada pelo consumo de alimentos extrainstitucionais e positivamente influenciada pelas ações de Educação Alimentar e Nutricional.

Palavras-chave Políticas públicas, Avaliação de programas e projetos de saúde, Alimentação escolar, Estudantes 


\section{Introdução}

A alimentação e a nutrição constituem requisitos básicos para a promoção e a proteção da saúde, possibilitando o pleno potencial de crescimento e desenvolvimento humano, com qualidade de vida e cidadania. Esses atributos estão consignados na Declaração Universal dos Direitos Humanos, cabendo ao Estado respeitar, proteger e facilitar a ação de indivíduos e comunidades em busca da capacidade de alimentar-se de forma digna ${ }^{1}$. Neste sentido, o Brasil tem desenvolvido um conjunto de políticas de Estado voltadas à concretização do direito humano à alimentação e nutrição adequadas, dentre elas, o Programa Nacional de Alimentação Escolar (PNAE).

O PNAE tem como diretrizes: o emprego da alimentação saudável e adequada; a inclusão da educação alimentar e nutricional no processo de ensino e aprendizagem; a descentralização das ações e articulação, em regime de colaboração, entre as esferas de governo; e o apoio ao desenvolvimento sustentável ${ }^{2}$.

O emprego da alimentação saudável deve compreender o uso de alimentos variados, seguros, que respeitem a cultura, as tradições e os hábitos alimentares saudáveis, contribuindo para o crescimento e o desenvolvimento dos alunos e para a melhoria do rendimento escolar, em conformidade com sua faixa etária e seu estado de saúde, inclusive dos que necessitam de atenção específica ${ }^{2}$.

No contexto da promoção da saúde, a escola apresenta-se como local de relevância para a adoção desta prática, atingindo grande parte da população, a baixo custo, por meio de uma estrutura organizada, que possibilita a formação de cidadãos críticos, com autonomia para o controle das suas condições de saúde e qualidade de vida ${ }^{3,4}$.

Assim, o presente estudo objetivou analisar o Programa de Alimentação Escolar sob a ótica dos alunos atendidos na rede estadual de ensino de Minas Gerais e, por conseguinte, o papel do Estado dentro deste programa, de modo a subsidiar melhorias e adequar o seu atendimento às características dos beneficiários.

\section{Métodos}

Realizou-se estudo transversal híbrido, de natureza quali-quantitativa, baseado em uma amostra representativa dos alunos da rede estadual de ensino de Minas Gerais, durante o período de agosto a novembro de 2010.
O estado de Minas Gerais possui a segunda maior rede de educação básica do país, organizada em 46 Superintendências Regionais de Ensino $(\mathrm{SRE})^{5}$.

Do universo de 2.291.780 alunos da rede estadual, foi calculada amostra no programa estatístico Stata SE (versão 12.0), levando-se em consideração efeito de desenho de 2,5 , precisão esperada de $4 \%$ e, por falta de estudos no estado sobre o tema, optou-se pela adoção, como referência, de prevalência esperada de 50\% dos escolares com a opinião de inadequação da Alimentação Escolar (AE), o que produziu uma amostra de 1500 alunos. Em seguida, considerando-se a proporcionalidade numérica dos alunos de cada SRE em relação ao universo da rede, foi realizada a distribuição da amostra por SRE.

Foram incluídos no estudo alunos matriculados no Ensino Fundamental (EF), Ensino Médio (EM) e Educação de Jovens e Adultos (EJA), respeitando-se a proporcionalidade que assumem no universo da rede estadual. Em cada regional de ensino foi sorteada aleatoriamente uma escola que atendesse simultaneamente aos três níveis acima descritos, na qual foram selecionados, também aleatoriamente, os alunos integrantes da amostra por SRE. Como critério de exclusão, as escolas que não atendiam a esta característica foram eliminadas do sorteio.

As escolas foram contactadas e os membros da direção esclarecidos sobre a finalidade da pesquisa. Os pais ou responsáveis receberam o Termo de Consentimento Livre e Esclarecido (TCLE) para autorização de participação dos estudantes na pesquisa.

Foi aplicado aos alunos questionário semiestruturado contemplando as seguintes categorias de estudo: 1) Consumo alimentar do aluno na escola, levando-se em consideração as possíveis fontes de obtenção dos alimentos: própria escola (PNAE; cantina ou venda escolar), entorno da escola (ambulantes; estabelecimentos próximos), ambiente familiar (moradia do aluno); 2) Abordagem dos temas alimentação e nutrição por meio de atividades educativas no ambiente escolar; 3) Adequação do espaço físico destinado ao consumo da AE; e 4) Percepção do aluno quanto à qualidade da alimentação ofertada.

Os participantes foram sorteados, organizados pelos professores e orientados por pesquisadores treinados no preenchimento individual dos questionários.

$\mathrm{Na}$ análise das perguntas fechadas e quantitativas, foram calculadas medidas descritivas (frequências absolutas e percentuais, média e desvio 
padrão) e inferenciais (teste qui-quadrado de Pearson ou teste exato de Fisher; $p<0,05$ ). Nas perguntas em que as variáveis explicativas (independentes) ofereciam mais de duas categorizações, para avaliar e localizar a diferença entre os grupos foi utilizado o qui-quadrado de partição.

As respostas às perguntas abertas foram categorizadas, a partir do conceito central contido na resposta, e analisadas por meio de frequência simples. Além disso, como foi permitido ao aluno fazer referência a mais de uma resposta, foi considerada a frequência com que cada resposta foi citada e, por conseguinte, criada uma nova variável-resposta.

Para avaliar a correlação entre as variáveis dependentes, previamente dicotomizadas, e as independentes, utilizou-se a regressão logística, obtendo-se a Odds Ratio e o respectivo intervalo de confiança a 95\%, p < 0,05.

Para a avaliação da qualidade da AE foram consideradas, entre outras variáveis, a aceitação e a adesão do aluno às refeições servidas. A adesão foi avaliada segundo os critérios de Bleil et al. ${ }^{6} \mathrm{e}$ Sturion et al. ${ }^{7}$, sendo considerada efetiva adesão ao programa o consumo igual ou superior a quatros vezes por semana. A aceitação foi avaliada por meio do percentual de alunos que referiram gostar em maior ou menor intensidade da alimentação oferecida, sendo considerada como efetiva aceitação as referências: "Ótima” ou "Muito Boa".

Os questionários foram duplamente digitados no software EpiData (versão 3.1), e a análise de consistência das informações e da estatística procedeu-se no software Stata SE (versão 12.0).

O projeto de pesquisa foi apreciado e aprovado pelo Comitê de Ética em Pesquisa da Universidade Federal de Ouro Preto.

\section{Resultados}

Participaram do estudo 1500 alunos da rede estadual de ensino. Destes, 1448 (96,5\%) forneceram informações consideradas válidas para o estudo.

Dos 1448 alunos, 896 (61,9\%) pertenciam ao Ensino Fundamental, $425(29,3 \%)$ ao Ensino Médio e 127 (8,8\%) à Educação de Jovens e Adultos. Do total de alunos, $842(58,1 \%)$ eram do sexo feminino e 606 (41,9\%) do sexo masculino.

Com relação às refeições produzidas e servidas pela escola, 7,5\% dos alunos referiram não consumi-las, $44,8 \%$ às vezes e $47,7 \%$ sempre.

A frequência de aparecimento de alimentos/ preparações nos cardápios escolares, segundo o relato dos alunos, é descrita na Tabela 1.

O consumo de alimentos não provenientes do PNAE foi referido por $83,5 \%$ dos alunos, sendo que, $13,2 \%$ dos alunos relataram levá-los de casa; $15,1 \%$ comprá-los de pessoas na escola; 19,5\% comprá-los de ambulantes; $24,7 \%$ comprá-los na cantina escolar; e 37,5\% comprá-los em locais próximos à escola.

Os alunos relataram levar de casa para consumo na escola principalmente frutas $(11,2 \%)$, guloseimas $(14,4 \%)$ e biscoitos $(26,1 \%)$, que representaram juntos $51,7 \%$ do total de alimentos relatados. Os refrigerantes e sucos representaram, respectivamente, $7,0 \%$ e $8,4 \%$.

Quanto ao espaço físico destinado ao consumo da AE, 28,0\% dos alunos afirmaram não ter na escola local específico para a realização das refeições. Entre os que afirmaram ter tal espaço, $40,1 \%$ não o utilizam, tendo como principal motivo (64,3\% dos alunos) a não adequação entre demanda e espaço físico.

Tabela 1. Frequência (\%) de aparecimento de alimentos no cardápio das escolas estaduais de Minas Gerais segundo o relato dos alunos $(\mathrm{n}=1448), 2010$.

\begin{tabular}{lcccccc}
\hline Alimento / Preparação & Não Aparece & 1x semana & 2x semana & 3x semana & 4x semana & 5x semana \\
\hline Fruta & 58.4 & 29.3 & 5.4 & 3.9 & 1.6 & 1.4 \\
Verdura & 19.8 & 23.6 & 24.6 & 18.0 & 7.5 & 6.5 \\
Legumes & 17.2 & 25.0 & 22.0 & 19.8 & 8.7 & 7.3 \\
Arroz & 8.9 & 14.6 & 24.3 & 20.9 & 19.5 & 11.8 \\
Feijão & 10.4 & 32.3 & 21.8 & 13.9 & 12.7 & 8.9 \\
Carnes & 15.7 & 25.8 & 22.9 & 14.1 & 8.8 & 12.7 \\
Macarrão & 11.5 & 48.2 & 26.1 & 10.2 & 2.7 & 1.3 \\
Farofa & 41.9 & 49.4 & 6.0 & 1.8 & 0.4 & 0.5 \\
Leite & 51.9 & 38.0 & 7.2 & 0.8 & 0.3 & 1.8 \\
Pão/Biscoito & 53.1 & 41.4 & 2.8 & 1.0 & 0.3 & 1.4 \\
Suco & 51.3 & 36.5 & 8.4 & 1.7 & 0.2 & 1.9 \\
Doces & 66.3 & 24.9 & 5.8 & 1.6 & 0.6 & 0.8 \\
\hline
\end{tabular}


A abordagem dos temas alimentação e nutrição no ambiente escolar foi relatada por $45,8 \%$ dos alunos. As seguintes atividades foram referidas: aulas práticas na horta escolar $(7,5 \%)$, aulas e palestras em sala de aula $(32,9 \%)$ e feira de ciências $(59,6 \%)$.

No que diz respeito às condições de atenção à saúde do escolar, 70,1\% dos alunos disseram nunca terem passado por uma avaliação física. Ainda sobre este aspecto, 5,6\% dos alunos referiram apresentar algum problema de saúde que configure necessidade alimentar especial. Do total de alunos, 90,3\% relatou que a escola não serve cardápio diferenciado para alunos com tais necessidades.

Sobre a qualidade da AE, 16,8\% dos alunos a consideravam ótima, $12,0 \%$ muito boa, $28,3 \%$ boa, $33,8 \%$ regular e $9,1 \%$ ruim. Dentre os que a consideravam regular ou ruim, um dos principais motivos alegados foi a "monotonia do cardápio", caracterizada pela repetição das preparações "macarrão" ou "sopa".

Avaliando a aceitação e a adesão dos alunos à AE, foi observado que $28,8 \%$ apresentaram efetiva aceitação e 45,1\% efetiva adesão ao programa.

As Tabelas 2 e 3 mostram os modelos finais de aceitação e adesão à $\mathrm{AE}$, respectivamente, e as variáveis independentes que se associaram a elas, contendo os valores brutos e ajustados de Odds Ratio.
A partir do modelo final da regressão logística, observa-se, pelos valores ajustados das Odds Ratio, que os homens apresentaram uma chance 1,35 vezes maior de aceitação da $\mathrm{AE}$ quando comparados às mulheres.

Em relação ao grupo etário, os alunos mais velhos (a partir de 15 anos), apresentaram uma chance de aceitação da $\mathrm{AE}$ 1,31 vezes maior que os mais jovens.

Quando se considerou a compra para consumo de alimentos extrainstitucionais, os alunos que não compram estes alimentos apresentaram o dobro de chance de aceitação da AE.

Considerando a participação em atividades de Educação Alimentar e Nutricional (EAN), os alunos que relataram participar destas atividades apresentaram aceitação 1,87 vezes maior da $\mathrm{AE}$.

Em relação ao modelo final da adesão à $\mathrm{AE}$, os alunos que participam de alguma atividade de EAN, apresentaram uma chance de adesão da ordem de 1,39 vezes maior quando comparados com seus colegas que não participam. Os alunos mais velhos (a partir de 15 anos) apresentaram uma chance 1,63 vezes maior de adesão quando comparados aos mais jovens.

Melhorias para a AE foram sugeridas por $73,5 \%$ dos alunos (Tabela 4 ).

Finalmente, as variáveis adesão e aceitação foram comparadas entre EF, EM e EJA (Tabela

Tabela 2. Modelo final de aceitação da Alimentação Escolar e variáveis independentes que se associaram a ela entre os alunos da rede pública estadual de MG, 2010.

\begin{tabular}{lcccc}
\hline \multicolumn{1}{c}{ Variável } & Odds Ratio Bruta & IC (95\%) & Odds Ratio Ajustada & IC (95\%) \\
\hline Sexo $^{*}$ & 1,36 & $1,0765-1,7144$ & 1,35 & $1,0720-1,7399$ \\
Grupo etário $^{* *}$ & 1,31 & $1,0314-1,6784$ & 1,31 & $1,1119-1,9067$ \\
$\begin{array}{l}\text { Consumo de alimentos } \\
\text { extra-institucionais }\end{array}$ & 2,05 & $1,5340-2,7567$ & 2,05 & $1,4828-2,8132$ \\
Atividades de EAN & & & & 1,87 \\
\hline
\end{tabular}

Valor de $\mathrm{p}<0,05$ e Log da verossimilhança $=-785,72529 .{ }^{*}$ Aceitação 1,35 vezes maior entre alunos do sexo masculino.

${ }^{* *}$ Aceitação 1,31 vezes maior entre alunos com idade superior a 15 anos. ${ }^{* * *}$ Aceitação 2,05 vezes maior entre alunos que não consumiam alimentos extra-institucionais. ${ }^{* * *}$ Aceitação 1,878 vezes maior entre alunos que participavam de atividades de EAN.

Tabela 3. Modelo final de adesão à Alimentação Escolar e variáveis independentes que se associaram a ela entre os alunos da rede pública estadual de MG, 2010.

\begin{tabular}{lcccc}
\hline \multicolumn{1}{c}{ Variável } & Odds Ratio Bruta & IC (95\%) & Odds Ratio Ajustada & IC (95\%) \\
\hline Grupo etário $^{*}$ & 1,51 & $1,2013-1,9021$ & 1,63 & $1,2849-2,0792$ \\
Atividades de EAN $^{* *}$ & 1,27 & $1,0165-1,5788$ & 1,39 & $1,1140-1,7549$ \\
\hline
\end{tabular}

Valor de $\mathrm{p}<0,05$ e Log da verossimilhança $=-905.10584$. * Adesão 1,63 vezes maior entre alunos com idade superior a 15 anos. ${ }^{* *}$ Adesão 1,39 vezes maior entre alunos que participavam de atividades de EAN. 
5). Observou-se que a adesão é significativamente maior ( $p=0,000)$ entre os estudantes do EJA $(72,9 \%)$ quando comparados aos do Ensino Médio (44,2\%) e Fundamental (41,2\%). A aceitação não apresentou diferença significativa entre os níveis de ensino.

\section{Discussão}

Os resultados mostraram baixa aceitação $(28,8 \%)$ e adesão $(45,1 \%)$ ao Programa. No geral, os estudos voltados à avaliação do PNAE demonstram adesão ao PNAE relativamente bai$\mathrm{xa}$, oscilando entre $38 \%$ e $62 \%{ }^{7-9}$.

Os principais desafios envolvidos na ampliação da adesão/aceitação das refeições perpassam pelo adequado planejamento do cardápio, que cabe estritamente ao profissional nutricionista, devidamente habilitado, conforme determina a Lei Federal no11947/2009: a responsabilidade técnica pela alimentação escolar nos Estados e Municípios caberá ao nutricionista responsável ${ }^{2}$.
Em Minas Gerais, configura-se situação extremamente precária no que diz respeito à disponibilização de profissionais para gestão e execução do PNAE. A Secretaria de Estado da Educação de Mina Gerais (SEE-MG) disponibiliza somente seis nutricionistas para atendimento a todo o estado, com 3.818 unidades escolares e mais de 2 milhões de alunos, o que inviabiliza o cumprimento das determinações da referida Lei ${ }^{2}$.

Segundo a maioria $(58,4 \%)$ dos alunos entrevistados, frutas não fazem parte do cardápio escolar. Entre as sugestões de melhoria para a $\mathrm{AE}$, a inclusão de frutas no cardápio é a que apresenta maior frequência (27\%) de citação pelos alunos.

O consumo no ambiente escolar de alimentos não provenientes do PNAE foi relatado por 83,5\% dos alunos, sendo a compra nas cantinas escolares e em locais próximos à escola as principais fontes de obtenção dos produtos, o que corrobora com os dados de Danelon et al. ${ }^{8}$ e Zancul ${ }^{10}$.

Foi observada aceitação significativamente maior entre os alunos que apresentaram menor

Tabela 4. Frequência de sugestões de melhoria feitas pelos alunos da rede estadual de ensino de Minas Gerais $(\mathrm{n}=1060), 2010$.

\begin{tabular}{lcc}
\hline \multicolumn{1}{c}{ Sugestões } & Frequência Absoluta & Frequência * $^{*}$ \\
\hline Incluir frutas no cardápio & 243 & 27,2 \\
Variar o cardápio & 219 & 24,5 \\
Incluir suco no cardápio & 130 & 14,6 \\
Incluir verduras no cardápio & 113 & 12,7 \\
Diminuir canjiquinha, macarrão e sopa no cardápio & 55 & 6,2 \\
Incluir legumes no cardápio & 53 & 5,9 \\
Tornar a alimentação mais saudável & 51 & 5,7 \\
Incluir guloseimas no cardápio & 50 & 5,6 \\
Melhorar o tempero & 45 & 5,1 \\
Melhorar a higienização & 37 & 4,2 \\
Incluir carnes no cardápio & 28 & 3,1 \\
Melhorar o porcionamento & 28 & 3,1 \\
Melhorar o humor das cozinheiras & 8 & 0,9 \\
\hline
\end{tabular}

*Obs.: Alguns alunos fizeram menção a mais de uma sugestão de melhoria.

Tabela 5. Comparação das frequências percentuais de adesão e aceitação à Alimentação Escolar entre os níveis de ensino da rede pública estadual de Minas Gerais, 2010.

\begin{tabular}{lccccc}
\hline & \multicolumn{3}{c}{ Modalidades de Ensino } & & \\
\cline { 2 - 5 } \multicolumn{1}{c}{ Variáveis } & Fundamental & Médio & EJA & $\boldsymbol{p}$ & $\chi^{2}$ \\
\hline Adesão & $41,24^{*}$ & $44,25^{*}$ & $72,95^{* *}$ & 0,000 & 43,12 \\
Aceitação & $27,25^{*}$ & $31,64^{*}$ & $29,84^{*}$ & 0.258 & 2,71 \\
\hline
\end{tabular}

Testes Qui-quadrado de Pearson e Qui-quadrado de Partição. * Não apresentou diferença estatística significativa. ${ }^{* *}$ Apresentou diferença estatística significativa. 
consumo de alimentos extrainstitucionais comprados. Hernández et al. ${ }^{11}$, em 2008, estudando o consumo da alimentação escolar por 1.398 alunos da rede pública, verificou que a $\mathrm{AE}$ é pouco consumida (41,8\% dos alunos) e que o consumo de alimentos externos colabora para este perfil.

Como forma de controlar a qualidade nutricional dos alimentos ofertados pelas cantinas escolares, alguns estados e municípios instituíram legislações específicas ${ }^{12,13}$.

Os alimentos levados de casa pelos alunos não apresentaram perfil diferente daquele consumido na cantina e no entorno escolar. As preferências alimentares dos alunos traduzem o percentual de $20,2 \%$ das sugestões de melhorias para a $\mathrm{AE}$, no qual são solicitadas inclusão de guloseimas $(5,6 \%)$ e sucos $(14,6 \%)$. No estudo realizado por Sturion et al. ${ }^{7}$, em 2005, avaliando fatores condicionantes da adesão ao Programa de Alimentação Escolar em escolas públicas, os escolares sugeriram como melhoria, o aumento da quantidade de carne e a oferta de suco.

A grande maioria (70\%) das sugestões de melhoria diz respeito à variação e à inserção de mais frutas, verduras e legumes no cardápio. $\mathrm{Na}$ rede estadual é comum a adoção de um cardápio semanal que é reproduzido ao longo do ano. Dessa forma, o aluno sabe, antecipada e repetidamente, qual preparação irá ser servida em cada um dos dias da semana.

Cerca de 50\% dos alunos referiram serem desenvolvidas em sua escola atividades educativas voltadas ao exercício dos temas alimentação e nutrição, sendo as principais mencionadas: fei- ra de ciências, aulas e palestras. Estudos de intervenção educativa no campo da nutrição constataram melhora na escolha alimentar dos estudantes e na atitude dos professores em relação à prevenção de hábitos alimentares inadequados ${ }^{14-}$ ${ }^{18}$. Porém, existe na literatura extenso debate sobre qual seria o real papel da EAN e qual a metodologia mais adequada a ser empregada ${ }^{10,19,20}$.

\section{Conclusão}

Os resultados apresentados levantam a necessidade da ampliação da adesão e aceitação do Programa pelos alunos, o que perpassa, dentre outras importantes ações inerentes à atuação do nutricionista, pelo adequado planejamento dos cardápios escolares e, consequentemente, pela garantia de quadro técnico suficiente para atendimento a todos os alunos da rede estadual. Ademais, sem considerar o tempo de permanência nas creches públicas, a grande maioria dos escolares fica exposta, em média, 12 anos a uma prática alimentar e nutricional pública que, dependendo da sua efetividade, poderá contribuir positiva ou negativamente para o processo de transição nutricional e de saúde que atualmente experimentamos. Desta forma, a atuação da Secretaria de Estado da Educação precisa ser revista e ações voltadas à melhoria da execução do programa devem ser implementadas, especialmente no que diz respeito à inserção da educação nutricional no contexto escolar dentro de uma proposta pedagógica.

\section{Colaboradores}

CAM Silva e LA Marques participaram da coleta dos dados, concepção, desenho, análise, interpretação e redação. E Bonomo, OMPA Bezerra e MS Corrêa participaram da interpretação e revisão do artigo. LSF Passos participou da coleta dos dados, concepção e desenho. AA Souza participou da coleta dos dados e revisão da literatura. BF Barros e DMS Souza participaram da aplicação estudo piloto, adaptação dos instrumentos e revisão da literatura. JA Reis e NG Andrade participaram da coleta dos dados, desenho e revisão do artigo. 


\section{Referências}

1. Brasil. Ministério da Saúde (MS). Secretaria de Atenção à Saúde. Política Nacional de Alimentação e Nutrição. Brasília: MS; 2003.

2. Brasil. Lei no 11.947 de 16 de junho de 2009. Dispõe sobre o atendimento da alimentação escolar e do Programa Dinheiro Direto na Escola aos alunos da educação básica. Diário Oficial da União 2009; 17 jun.

3. Félix DS. Obesidade. In: Teixeira Neto F. Nutrição Clínica. Rio de Janeiro: Guanabara Koogan; 2003. p. 185-195.

4. Brasil. Ministério da Saúde (MS), Organização Panamericana da Saúde. Secretaria de Gestão do Trabalho e da Educação na Saúde. Escolas promotoras de saúde: experiências do Brasil. Brasília: MS; 2006.

5. Secretaria de Estado da Educação de Minas Gerais. Lista de superintendências. Belo Horizonte: SEE; 2008. [atualizado em 2011]. [acessado 2011 out 04]. Disponível em: https://www.educacao.mg.gov.br/

6. Bleil RAT, Salay E, Silva MV. Adesão ao programa de alimentação escolar por alunos de instituições públicas de ensino no município de Toledo, PR. Rev Segurança Alimentar e Nutricional 2009; 16(Supl. 1):65-82.

7. Sturion GL, Silva MV, Ometto AMH, Furtuoso MCO, Pipitone MAP. Fatores condicionantes da adesão dos alunos ao Programa de Alimentação Escolar no Brasil. Rev Nutr 2005; 18(2):167-181.

8. Danelon MS, Fonseca MCP, Silva MV. Preferências alimentares no ambiente escolar. Rev Segurança Alimentar e Nutricional 2008; 15(Supl. 2):66-84.

9. Pegolo GE, Silva MV. Consumo de energia e nutrientes e a adesão ao Programa Nacional de Alimentação Escolar (PNAE) por escolares de um município paulista. Rev Segurança Alimentar e Nutricional 2010; 17(Supl. 2):50-62.

10. Zancul MS. Consumo alimentar de alunos nas escolas de ensino fundamental em Ribeirão Preto (SP) [dissertação]. Ribeirão Preto (SP): Universidade de São Paulo, Departamento de Medicina Social, Faculdade de Medicina de Ribeirão Preto; 2004.

11. Hernández AB, Slavutzky SMB, Padilha DMP. Avaliação do consumo da merenda escolar em escolas municipais de Porto Alegre. Rev Fac Odontol 2008; 49(Supl. 1):26-30.

12. Willhelm FF, Ruiz E, Oliveira AB. Cantina escolar: qualidade nutricional e adequação à legislação vigente. Rev HCPA 2010; 30(Supl. 3):266-270.
13. Brasil. Lei no 15.072 de 05 de abril de 2004. Dispõe sobre a promoção da educação alimentar e nutricional nas escolas públicas e privadas do sistema estadual de ensino. Diário Oficial da União 2004; 05 abr.

14. Gaglianone CP, Taddei JAAC, Colugnati FAB, Magalhães CG, Davanço GM, Macedo L, Lopez FA. Educação nutricional no ensino público fundamental em São Paulo, Brasil: projeto redução dos riscos de adoecer e morrer na maturidade. Rev Nutr 2006; 19(3):309-320.

15. Rodrigues LPF, Roncada MJ. Educação nutricional no Brasil: evolução e descrição de proposta metodológica para escolas. Comun Ciênc Saúde 2008; 19(Supl. 4):315-322.

16. Davanço GM, Taddei JAAC, Gaglianone CP. Conhecimentos, atitudes e práticas de professores de ciclo básico, expostos e não expostos a curso de educação nutricional. Rev Nutr 2004; 17(2):177-184.

17. Vargas ICS, Sichieri R, Sandre-Pereira G, Veiga GV. Avaliação de programa de prevenção de obesidade em adolescentes de escolas públicas. Rev Saude Publica 2011; 45(1):59-68.

18. Gabriel CG, Santos MV, Vasconcelos FAG. Avaliação de um programa para promoção de hábitos alimentares saudáveis em escolares de Florianópolis, Santa Catarina, Brasil. Rev Bras Saude Mater Infant 2008; 8(3):299-308.

19. Domene SMA. A escola como ambiente de promoção da saúde e educação nutricional. Psicol 2008; 19(Supl. 4):505-517.

20. Toral N, Conti MA, Slater B. A alimentação saudável na ótica dos adolescentes: percepções e barreiras à sua implementação e características esperadas em materiais educativos. Cad Saude Publica 2009; 25(11):2386-2394.

Artigo apresentado em 10/10/2012

Aprovado em 16/11/2012

Versão final apresentada em 19/12/2012 
